Recepción: 20 / 12 / 2017

Aceptación: 15 / 02 / 2018

Publicación: 8 / 05 / 2018

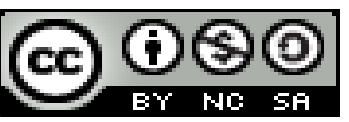

Ciencias Químicas

Artículo de Revisión

\title{
Elaboración de pan con harina de trigo, enriquecido con harina de soya y fibra soluble para mejorar su valor nutritivo
}

Preparation of bread with wheat flour, enriched with soy flour and soluble fiber to improve its nutritional value

Panificação com farinha de trigo, enriquecida com farinha de soja e fibra solúvel para melhorar seu valor nutricional

Carlos J. Silva-Huilcapi ${ }^{\text {I }}$

carlos.silvah@ug.edu.ec

Haydee M. Alvarado-Alvarado II

haydee.alvaradoa@ug.edu.ec

Liliana A. Cortez-Suarez III

lcortez@utmachala.edu.ec

Walter E. Mariscal-Santi IV

walter.mariscals@ug.edu.ec

Zoila B. Luna-Estrella V

zoila.lunae@ug.edu.ec

Correspondencia: carlos.silvah@ug.edu.ec

\footnotetext{
I Docente, Universidad de Guayaquil, Guayaquil, Ecuador.

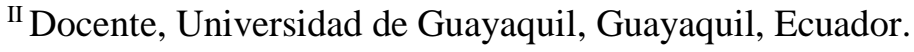

III Docente, Universidad de Guayaquil, Guayaquil, Ecuador.

${ }^{\text {IV }}$ Docente, Universidad de Guayaquil, Guayaquil, Ecuador.

${ }^{\mathrm{v}}$ Docente, Universidad de Guayaquil, Guayaquil, Ecuador.
} 


\section{Resumen}

En el presente estudio se da a conocer las condiciones de proceso y las características de un pan el cual se encuentra elaborado a base de harina de trigo, parcialmente sustituida con harina de soya y fibra soluble adicionada. Se trata de un estudio experimental el cual se aplica principalmente al momento del desarrollo del prototipo, cuando se pretende determinar las cantidades adecuadas de sustitución en la harina de trigo por harina de soya, para lo cual se llevaron a cabo cuatro experimentos. Asimismo, es un estudio cualitativo, donde se aplicó la revisión bibliográfica para poder elaborar un concepto del diseño del producto deseado y caracterizar el producto final. Durante el desarrollo del producto se aplicó la metodología cuantitativa para la tabulación de resultados en el desarrollo experimental y de la fase final durante la evaluación sensorial del producto. Por último, es importante destacar que el propósito del estudio se centra en la elaboración de un producto mejorado nutricionalmente con aporte de la proteína con aminoácidos esenciales de la soya y fibra soluble añadida, para mejorar la digestión de los consumidores, como una alternativa de consumo al pan tradicional elaborado cien por ciento con harina de trigo.

Palabras clave: Harina, trigo, soya, fibra, sustitución. 
Carlos J. Silva-Huilcapi; Haydee M. Alvarado-Alvarado; Liliana A. Cortez-Suarez; Walter E. Mariscal-Santi; Zoila B. LunaEstrella

\begin{abstract}
In the present study, the process conditions and the characteristics of a bread which is elaborated based on wheat flour, partially substituted with soy flour and soluble fiber added, is made known. This is an experimental study which is mainly applied at the time of the development of the prototype, when it is intended to determine the adequate amounts of substitution in wheat flour for soybean meal, for which four experiments were carried out. Likewise, it is a qualitative study, where the bibliographic review was applied to be able to elaborate a design concept of the desired product and characterize the final product. During the development of the product, the quantitative methodology was applied for the tabulation of results in the experimental development and the final phase during the sensory evaluation of the product. Finally, it is important to emphasize that the purpose of the study is focused on the elaboration of a nutritionally enhanced product with the contribution of the protein with essential amino acids from soy and added soluble fiber, to improve the digestion of consumers, as a consumption alternative Traditional bread made one hundred percent with wheat flour.
\end{abstract}

Keywords: Flour, wheat, soy, fiber, substitution. 


\section{Introducción.}

El pan es uno de los alimentos básicos de la dieta humana formando parte de la alimentación de muchas civilizaciones debido a sus características nutritivas y a la sencillez de aplicación culinaria de la materia prima.

Los cereales pertenecen a la familia de las gramíneas. Entre los más usados en la elaboración de varios alimentos y en especial en la fabricación de pan, se encuentra el trigo, que como alimento empezó a usarse desde la antigüedad en la fabricación de pan debido a su gran cantidad de gluten y a su composición, dejando atrás varios cereales como el maíz, el arroz, etc.

El trigo es uno de los cereales más versátiles, y además, es una de las gramíneas cuyo cultivo es mayor debido a que contiene los cinco nutrientes más importantes que el organismo necesita para su buen desarrollo y prevención de varias enfermedades, entre ellos los carbohidratos, minerales, vitaminas, grasas y proteínas.

De acuerdo con el Ministerio de Industrias y Productividad el 98.45\% del trigo consumido en el Ecuador llega a través de importación directa; existen varios programas de sustituciones parciales de harina de trigo con la finalidad de reducir las importaciones, según esta cartera, sustituyendo el 5\% en el país se ocasionaría un ahorro de 12.3 millones de dólares pues se dejaría de importar 32.0000 toneladas de harina.

Adicionalmente el Instituto de Promoción Exportaciones e Inversiones del Ecuador, PRO ECUADOR, indica que los consumos de pan con relación al tiempo van aumentando así por ejemplo en Alemania el consumo de pan aumentó considerablemente hasta alcanzar los $126 \mathrm{~kg}$, en el país de Chile el consumo alcanzó los 98 kg, mientras que en nuestro país alcanzó los 37 kg per 
Carlos J. Silva-Huilcapi; Haydee M. Alvarado-Alvarado; Liliana A. Cortez-Suarez; Walter E. Mariscal-Santi; Zoila B. LunaEstrella

cápita. Con respecto a los productos horneados en general en el Ecuador se registró un incremento del 5.7\% al año 2012 y se prevé que para el año 2018 el consumo se incremente hasta llegar a los 683 millones de dólares, ya que en el año 2012 el valor de consumo fue de US\$ 632 millones, lo que representa un aumento en porcentaje del $8 \%$.

Considerando la gran importancia del consumo del pan en los consumidores, el estudio pretende desarrollar una alternativa alimenticia (pan) sustituyendo un porcentaje determinado de harina de trigo por harina de soya, su valor proteico se ve incrementado lo cual determina a través de la experimentación y también de la fortificación añadiendo una cantidad considerable de fibra soluble que ayuda con la digestión de los futuros consumidores, del pan enriquecido con harina de soya y fibra soluble, beneficioso para la salud humana.

La harina de trigo es uno de los ingredientes principales en la elaboración de pan, sus principales componentes son: (Quaglia, 2008)

- Almidón 70-75\%

- Agua $14 \%$

- Proteínas $10-12 \%$

- Lípidos $2 \%$

La harina de trigo contiene gluten, siendo un complejo de proteínas insolubles en agua, del cual se derivan dos principales proteínas llamadas glutenina y gliadina.

- La glutenina es la proteína que le da fuerza y tenacidad a la masa (30-40\%) de las proteínas totales del trigo y se encuentran en la parte del albumen; solubles en soluciones ácidas (Romain, Thomas, Pierre, \& Gerard, 2010) 
- La gliadina es la proteína que le da elasticidad a la masa (40-50\%) de las proteínas totales del trigo se concentran básicamente en el endospermo y son solubles en soluciones alcohólicas (Romain, Thomas, Pierre, \& Gerard, 2010)

- Adicionalmente encontramos las albúminas y las globulinas, cada una representa entre el 5- 10\% de las proteínas totales del trigo respectivamente.

La soya Glycine max, es una de las mayores fuentes de proteínas, rica en ciertos componentes funcionales tales como las isoflavonas, fibras, flavonoides, terpenos, fitoesteroles y vitaminas como tiamina, niacina, riboflavina y B6; de alto valor en minerales como calcio, magnesio, zinc y hierro. (Bautista, 2007)

La harina de soya es un polvo fino que se obtiene tras el tostado y molido de las semillas, casi no contiene almidón, por lo que se usa para la elaboración de productos dietéticos. Contiene un $50 \%$ de proteínas, y ésta riqueza la hace idónea en las dietas destinadas para el consumo humano. Se emplea para enriquecer el valor proteico de muchas preparaciones.

Al añadirla a otras harinas obtenidas de cereales, esta aumenta el valor nutricional de las mismas al compensar su déficit en el aminoácido lisina. Su contenido en lecitina es elevado. (Calvo, 2003)

Entre las propiedades destacadas: (Criscaut, 2006)

- No contiene gluten.

- Realza la estabilidad de las vitaminas en la panificación.

- Compensa aminoácidos deficientes de la harina de trigo.

- Mejora el producto final en la elaboración de pan. 
Carlos J. Silva-Huilcapi; Haydee M. Alvarado-Alvarado; Liliana A. Cortez-Suarez; Walter E. Mariscal-Santi; Zoila B. LunaEstrella

- Obtención de una miga más suave.

- Mejora el balance bilógico del producto.

- Evita el uso de grandes cantidades de huevo.

La fibra siendo adicionada al pan, permite que este tenga un mejor producto final en su elaboración, ya que la fibra soluble potencializa las características propias de la harina de trigo, y otras harinas provenientes de cereales. (García, 2004)

La fibra soluble, tiene dos características esenciales en la panificación, brinda suavidad al pan, y alarga la vida de este.

En la actualidad, el trigo que se importa en el Ecuador proviene en su mayor parte de Canadá y Estados Unidos, sin embargo el gobierno está impulsando la reestructuración de la matriz productiva para que en la medida de lo posible se utilicen materias primas nacionales para la elaboración de productos con valor agregado así que según el plan que tiene el gobierno ecuatoriano en aumentar la producción nacional para poder disminuir los valores de importación de diversos productos y que puedan ser sustituidos por producción nacional .

De acuerdo con un reporte de la Organización Mundial para la Alimentación y la Agricultura (FAO, 2014) un estudio de cereales del período de 2004 a 2014 pasó de 2110 a 2530 millones de toneladas a nivel mundial, según este estudio la producción de trigo para el año 2015 es de 720 millones de toneladas.

A esto se puede sumar que la mayoría de productos existentes en el mercado son elaborados a base de trigo, existen ciertos panes en los cuales existe reducción de la harina de trigo por otros tipos de cereales pero no contienen aporte nutricional adecuado, según un informe de la Encuesta de 
Salud y Nutrición de Ecuador existe un 31.5 de porcentaje de la población de la Costa con desnutrición, a su vez el 19,1 del porcentaje presenta talla baja para las edades de cada ciudadano y un 6,1 de porcentaje de consumo inadecuado de proteínas y nutrientes a nivel nacional por lo que se denota la falta de información por parte de la ciudadanía en consumir alimentos de valor nutricionalmente adecuados.

\section{Metodología.}

Se basa en un estudio cuantitativo, es decir, un contraste de teorías ya existentes a partir de una serie de hipótesis surgidas de la misma, siendo necesario para obtener una muestra.

Este método utiliza la recolección y el análisis de datos para contestar preguntas de investigación, probar hipótesis establecidas, y confía en la medición numérica y el uso de la estadística representada por gráficos y tablas para establecer patrones exactos de comportamiento y probar teorías. Las conclusiones se establecen mediante este método.

Durante el desarrollo del producto se aplica esta metodología para la tabulación de resultados en el desarrollo experimental y de la fase final durante la evaluación sensorial del producto. (Rodríguez, 2013)

Asimismo, reúne el método Cualitativo, el cual consiste en la construcción de una teoría a partir de una serie de proposiciones extraídas de un cuerpo teórico. Los estudios mediante este método pueden desarrollar preguntas e hipótesis antes, durante o después de la recolección y análisis. 
Carlos J. Silva-Huilcapi; Haydee M. Alvarado-Alvarado; Liliana A. Cortez-Suarez; Walter E. Mariscal-Santi; Zoila B. LunaEstrella

La metodología cualitativa es inductiva, los investigadores desarrollan conceptos partiendo de pautas de datos y no recogiendo datos para evaluar modelos, hipótesis o teorías prefijadas. (Rodríguez, 2013)

Durante la revisión bibliográfica en la parte inicial de la experimentación y en la parte final de la misma se aplica la metodología cualitativa para poder elaborar un concepto del diseño del producto deseado y para poder caracterizar el producto final.

Por último, se utiliza el método de experimentación, conocido como una de los más eficaces y complejos, en el cual el investigador crea las condiciones necesarias para el esclarecimiento de las propiedades y relaciones del objeto de estudio que son de utilidad en la investigación.

Se aplica principalmente al momento del desarrollo del prototipo, al momento de determinar las cantidades adecuadas de sustitución en la harina de trigo por harina de soya.

Para la experimentación se obtiene como variable independiente la cantidad de harina de soya en sustitución parcial de harina de trigo. La cual es desarrollada en los talleres de panadería de la facultad de Ingeniería Química de la Universidad de Guayaquil, en los laboratorios AVVE y GRANOTEC.

Inicialmente se desarrolla la formulación de pan con $100 \%$ de harina de trigo siendo el prototipo del cual se va a realizar la sustitución.

Se llevaron a cabo cuatro experimentos, en los cuales, para el primero se llevó a cabo la fórmula de pan 100\% de harina de trigo y 3\% de fibra soluble, en el segundo experimento se realizó la formulación de pan con $90 \%$ de harina de trigo, $10 \%$ harina de soya, para el tercero la fórmula de 
pan con $85 \%$ de harina de trigo, $15 \%$ harina de soya, y para el cuarto la fórmula de pan con $80 \%$ de harina de trigo, $20 \%$ harina de soya y $3 \%$ de fibra soluble.

\section{Resultados.}

Determinación del volumen especifico de la formulación de los panes

Se realizó la determinación del pan de trigo, pan elaborado con $15 \%$ de harina de soya, y 20\% de harina de soya. En la formulación de pan con estos porcentajes, se comprobó cambios en el volumen específicos del producto final.

El volumen obtenido del pan de trigo es de $2.29 \mathrm{ml} / \mathrm{g}$. El volumen del pan con $15 \%$ de harina de soya es de $1.82 \% \mathrm{ml} / \mathrm{g}$.

En la fórmula del pan con $15 \%$ de harina de soya, hubo decrecimiento del volumen; con el pan de $20 \%$ de harina de soya su volumen decreció al igual que la fórmula del $15 \%$.

El pan que tiene menor volumen específico es el que tiene $20 \%$ de harina de soya, ya que, con el aumento de la harina de soya en reemplazo de la harina de trigo, disminuya el gluten, que es la proteína que da elasticidad y expansibilidad del pan.

\section{Análisis de resultados reológicos}

El índice de absorción se mantiene uniforme en todas las mezclas de harinas.

La harina de soya de 10\%, respecto a las características reológicos, es la que más se asemeja a la de trigo. 
Carlos J. Silva-Huilcapi; Haydee M. Alvarado-Alvarado; Liliana A. Cortez-Suarez; Walter E. Mariscal-Santi; Zoila B. LunaEstrella

La harina de soya al 15\%, se observa que al igual que el experimento anterior que el índice de absorción se mantiene uniforme en todas las mezclas de harinas, por otra parte se obtuvo que a mayor sustitución de harina de soya, decrecen los valores de amasado, gluten, viscosidad, amilasas y retrogradación.

Al 20\% el índice de absorción igualmente se mantiene uniforme en todas las mezclas de harinas y se observa que a mayor sustitución de harina de soya, decrecen los valores de amasado, gluten, viscosidad, amilasas y retrogradación.

Formulación final del pan de soya con fibra soluble

Tabla 1 Fórmula final del producto

\begin{tabular}{|c|c|c|c|}
\hline Ingredientes & Cantidad & Unidad & $\begin{array}{c}\text { Porcentaje } \\
\text { Panadero }\end{array}$ \\
\hline Harina de trigo & 800 & $\mathrm{~g}$ & $39,40 \%$ \\
\hline Harina de soya & 200 & $\mathrm{~g}$ & $9,85 \%$ \\
\hline Fibra Soluble & 30 & $\mathrm{~g}$ & $1,47 \%$ \\
\hline Azúcar & 44 & $\mathrm{~g}$ & $2,2 \%$ \\
\hline Huevos & 154 & $\mathrm{~g}$ & $7,5 \%$ \\
\hline Manteca & 146 & $\mathrm{~g}$ & $7,1 \%$ \\
\hline Levadura & 62 & $\mathrm{~g}$ & $3,0 \%$ \\
\hline Agua & 580 & $\mathrm{~g}$ & $28,57 \%$ \\
\hline Sal & 14 & $\mathrm{~g}$ & $0,7 \%$ \\
\hline Total & $\mathbf{2 0 3 0}$ & $\mathbf{g}$ & $\mathbf{1 0 0 \%}$ \\
\hline
\end{tabular}

Fuente: El autor 


\section{Conclusiones}

- Luego de haber realizado la experimentación sustituyendo el 5\%, 10\%, 15\% y $20 \%$ de harina de trigo, la última formulación presentada presenta las mejores características, siendo el $20 \%$ de reemplazo la fórmula ganadora.

- El pan propuesto cumple con las características físicas y organolépticas de la norma INEN 530: 1980 Ensayo de Panificación, dentro del cual se evalúan los parámetros de: peso, volumen, apariencia y simetría, color de la miga, textura de la miga y grano de la miga.

- El pan cumple con los parámetros de humedad, cenizas, grasas, fibra y proteínas y la norma INEN toma como referencia la Norma Venezolana CONVENIN 226-88 primera revisión para requisitos de pan.

\section{Recomendaciones}

- Dentro de la formulación no se debe exceder porcentaje mayor al recomendado en este estudio pues las características del pan comienzan a cambiar las características organolépticas, principalmente la textura.

- Se deben garantizar las condiciones de inocuidad del producto, por el cual se recomienda la aplicación correcta de las Buenas Prácticas de Manufactura, durante la elaboración, almacenaje y distribución del pan.

- Este estudio puede ser utilizado como fuente bibliográfica inicial y de referencia para otros tipos de estudios similares de sustitución de harina de trigo o para estudios de implementación de negocios en este tipo de productos. 
Carlos J. Silva-Huilcapi; Haydee M. Alvarado-Alvarado; Liliana A. Cortez-Suarez; Walter E. Mariscal-Santi; Zoila B. LunaEstrella

\section{Bibliografía}

Bautista, J. (2007). Desarrollo de pan integral con soya, chia, linaza y acido folico como alimento funcional para la mujer. . Archivos norteamericanos de nutricion.

Calvo, D. (Mayo de 2003). www.diodora.com. Recuperado el 25 de abril de 2018, de http://www.diodora.com/documentos/nutricion_soja.htm

Criscaut, A. (2006). http://alimentos.org.es/harina-soj. Recuperado el 25 de abril de 2018, de http://alimentos.org.es/harina-soj: http://alimentos.org.es/harina-soj

Garcia, N. (2004). http://www.mimediconatural.com/beneficios-y-propiedades-de-harina-desoja/yfibra. Recuperado el 25 de abril de 2018, de http://www.mimediconatural.com/beneficios-y-propiedades-de-harina-de-soja/yfibra: http://www.mimediconatural.com/beneficios-y-propiedades-de-harina-de-soja/yfibra

Quaglia, G. (2008). Ciencia y tecnologia de la panificacion.

Rodríguez, P. (2013). Eumed.net. Recuperado el 26 de abril de 2018, de Eumed.net: http://www.eumed.net/tesis-doctorales/2012/eal/metodologia_cuant

Romain, Thomas, Pierre, \& Gerard. (2010). Ciencia de los Alimentos. Zaragoza: Acribia. 